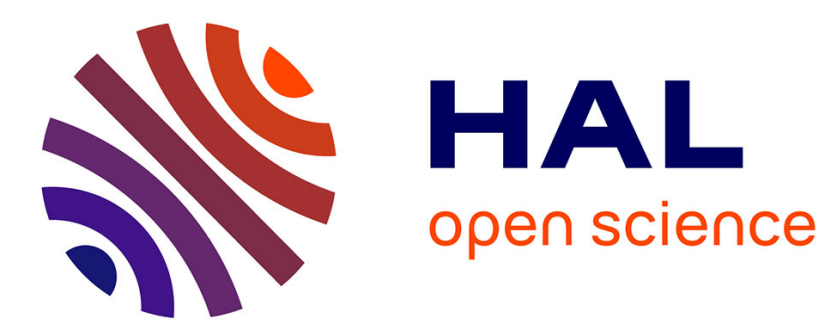

\title{
Pour une sociologie des usages et de l'innovation appliquée aux récits innovants
}

\author{
Julien Falgas
}

\section{To cite this version:}

Julien Falgas. Pour une sociologie des usages et de l'innovation appliquée aux récits innovants. Pascal Robert. Bande dessinée et numérique, CNRS Éd., pp.135-153, 2016, 978-2-271-08759-1. hal$01278659 \mathrm{v} 2$

\section{HAL Id: hal-01278659 \\ https://hal.science/hal-01278659v2}

Submitted on 16 Jun 2016

HAL is a multi-disciplinary open access archive for the deposit and dissemination of scientific research documents, whether they are published or not. The documents may come from teaching and research institutions in France or abroad, or from public or private research centers.
L'archive ouverte pluridisciplinaire HAL, est destinée au dépôt et à la diffusion de documents scientifiques de niveau recherche, publiés ou non, émanant des établissements d'enseignement et de recherche français ou étrangers, des laboratoires publics ou privés.

$$
\text { Copyright }
$$




\section{Pour une sociologie des usages et de l'innovation appliquée aux récits innovants}

Julien Falgas

Considérons l'acte de raconter une histoire comme un acte de communication. Cet acte de communication s'inscrit dans un contexte sociotechnique particulier. Plutôt que d'étudier les caractéristiques formelles du récit - objet par lequel s'incarne l'acte de communication - il s'agira ici d'éclaircir ce qui - dans le contexte sociotechnique - concourt à l'adoption d'une forme singulière de récit, par les auteurs et par leurs lecteurs.

L'ère numérique se caractérise par l'existence de technologies qui permettent à toutes les formes narratives traditionnelles de coexister sur les mêmes écrans. On peut aujourd'hui, sur le même ordinateur ou la même tablette tactile :

1. se nourrir indifféremment de littérature, de cinéma, de feuilletons télévisés ou radiophoniques ou encore de bandes dessinées.

2. accueillir conjointement des formes de publications plus ou moins narratives parmi lesquelles l'information journalistique, les pratiques vidéoludiques ou l'écoute musicale...

3. Mais aussi accéder à tout l'éventail des pratiques sociales et conversationnelles offertes par le courrier électronique, la messagerie instantanée, les forums de discussion, ou les réseaux sociaux numériques.

Postulons qu'un tel brassage des formes communicationnelles débouchera sur l'apparition et la stabilisation de formes nouvelles, comme à chaque occasion où de nouveaux outils de communication se sont répandus ${ }^{1}$. Sans préjuger de l'avenir de la bande dessinée à l'ère numérique, son héritage irrigue d'ores-et-déjà les évolutions en cours. Ce constat invite à souscrire à l'ambition de Lev Manovitch d'éclairer aujourd'hui les dynamiques par lesquelles naîtront les langages de demain ${ }^{2}$.

\section{Comment expliquer l'innovation narrative?}

Au fil de l'évolution de ce que l'on nomme aujourd'hui la bande dessinée numérique, tandis que les théoriciens cherchent à comprendre si les formes produites par quelques auteurs pionniers répondent à la définition sémiotique de la bande dessinée ${ }^{3}$, les professionnels se demandent quel modèle économique pourrait rendre de tels récits compatibles avec leur propre définition de la bande dessinée,

\footnotetext{
${ }^{1}$ Lire à ce sujet CLÉMENT, Jean, 2000. Hypertextes et mondes fictionnels ou l'avenir de la narration dans le cyberespace.

Disponible à l'adresse : http://archivesic.ccsd.cnrs.fr/sic_00000294/fr/.

${ }^{2}$ MANOVITCH, Lev, 2001. Le langage des nouveaux médias [en ligne]. 2010. trad. de l'anglais par R. Crevier, Dijon : les Presses du réel. ISBN 9782840663423.

${ }^{3}$ Préoccupation majeure qui sert de point de départ au chapitre consacré à la bande dessinée numérique dans GROENSTEEN, Thierry, 2011, Bande dessinée et narration, Système de la bande dessinée 2. Paris : P.U.F. Formes sémiotiques. ISBN 9782130584872.
} 
en tant qu'industrie culturelle. Pour sortir de l'ornière définitionnelle, effectuons un pas de côté au travers de l'exemple de deux récits publiés au cours des dernières années et explicitement affiliés au champ de la «bande dessinée numérique »:

1. Le premier, la série Les Autres Gens ${ }^{4}$,

a. se présente comme un feuilleton quotidien,

b. publié entre mars 2010 et juin 2012 sur le site web lesautresgens.com

c. sous forme d'épisodes d'une quarantaine de vignettes que le lecteur consulte successivement comme il le ferait pour un diaporama de photographies.

2. Le second récit, la série MediaEntity,

a. se veut un récit transmédia ${ }^{6}$ dont l'expression initiale a consisté en quatre épisodes hebdomadaires

b. publiés sur le site web mediaentity.net au cours du mois de décembre 2012,

c. sous forme de diaporama d'images qui pouvaient consister en des vignettes isolées ou des agencements plus complexes de plusieurs vignettes, organisées selon les canons habituels de la bande dessinée.

L'originalité formelle de ces récits par rapport à la bande dessinée traditionnelle réside dans le recours au diaporama. II ne s'agira pas d'approfondir ici les nombreuses subtilités formelles et langagières qui accompagnent ce glissement. II est toutefois primordial de relever son lien avec le format «turbomédia » inventé par Yves Bigerel sous le pseudonyme de Balak et promu par Alexandre Ulmann sous le pseudonyme de Malec ${ }^{7}$. Quel que soit l'avenir de ce format, il s'est imposé en quelques années comme un jalon formel des évolutions de nos langages narratifs à l'ère numérique, en convainquant lecteurs et auteurs de sa pertinence, mais aussi plusieurs grands noms de l'industrie culturelle de la bande dessinée au premier rang desquels l'éditeur américain Marvel qui a sollicité le conseil de Yves Bigerel dans le développement de récits destinés aux tablettes tactiles.

Si les caractéristiques formelles évidentes des deux récits étudiés suffisent à attester de leur caractère inventif - sinon innovant - leur existence soulève plusieurs questions :

- Comment de tels récits ont-ils pu être imaginés, produits, publiés et lus ?

- Comment leurs auteurs y sont-ils parvenus dans des conditions professionnelles, compatibles avec les contraintes économiques?

- Quelle est la pérennité de leurs inventions?

Pour le comprendre, il convenait de s'en remettre aux auteurs et aux lecteurs ${ }^{8}$. Notre ambition consiste ici à mettre en évidence ce que l'on appelle en sociologie les cadres de référence ${ }^{9}$ :

\footnotetext{
${ }^{4}$ CADENE et al., 2010, « Les Autres Gens ». Disponible à l'adresse : http://www.lesautresgens.com (consultée le 4 septembre 2015).

${ }^{5}$ Emilie et Simon, 2012, «MediaEntity », Disponible à l'adresse : http://www.mediaentity.net (consultée le 4 septembre 2015).

${ }^{6}$ Un récit transmédia est un récit-monde que les publics appréhendent de manière parcellaire en accédant à tout ou partie des formes narratives proposées. Le contact d'un univers fictionnel par le biais d'une pluralité de médias entraîne un effet de réel, du fait que notre appréhension de la réalité s'appuie elle-même pour bonne part sur sa perception au travers de ces mêmes médias (JENKINS, Henry, 2006. Convergence culture: where old and new media collide. New York : New York University Press. ACLS Humanities E-Book. ISBN 9780814742815).

${ }^{7}$ Une frise interactive retrace l'histoire de ce format narratif entre 2009 et 2014 : Mast et Geoffo, 2014, 5 ans de Turbomédia, disponible en ligne : http://ybrik-media.com/bon-anniversaire-le-turbomedia/ (consultée le 23 janvier 2015).

${ }^{8}$ La méthodologie, les résultats et l'ensemble de cette étude ont fait l'objet d'une thèse de doctorat : FALGAS, Julien, 2014.

Raconter à l'ère numérique : auteurs et lecteurs héritiers de la bande dessinée face aux nouveaux dispositifs de publication. Thèse
} 
FALGAS, Julien, 2016. Pour une Sociologie des usages et de l'innovation appliquée aux récits innovants.

In : ROBERT, Pascal, Bande dessinée et numérique. CNRS Editions, collection Les Essentiels d'Hermès. Paris. p. 135-153.

- auxquels les auteurs ont fait appel dans l'élaboration des récits et des dispositifs de publication,

- et ceux sollicités par les lecteurs pour aborder l'expérience offerte par ces récits et leur environnement de publication.

\section{Les Autres Gens, un assemblage original de cadres de référence}

L'étude a montré que la genèse du projet de cette série était intervenue au carrefour de quelques grands cadres de référence dont le partage réussi entre les auteurs et les lecteurs de la série a concouru à sa pérennité. Les auteurs de la série Les Autres Gens sont ainsi parvenus à proposer chaque jour, durant deux ans et demi, cinq minutes de lecture graphique aux souscripteurs d'un abonnement mensuel de trois euros.

La publication numérique, cadre de référence pour la forme plastique du récit

Le volume de contenu était si important que les auteurs n'envisageaient pas qu'une publication imprimée soit possible. C'est pourquoi ils ont imaginé leur projet comme exclusivement numérique. Quelques mois après le commencement de la série, l'éditeur Dupuis leur a pourtant offert une adaptation imprimée, grâce à laquelle l'équilibre économique du projet a pu être atteint. Malgré cela, les auteurs ont continué à concevoir leur récit selon sa forme initiale, sans anticiper l'adaptation imprimée. La forme choisie est celle d'un diaporama de vignettes. Ce choix a été effectué en référence aux usages en vigueur sur l'iPhone pour la lecture de bandes dessinées numérisées : début 2010, l'appareil d'Apple était le principal smartphone disponible sur le marché et la commercialisation de l'iPad annoncé fin janvier ne devait débuter que le 3 avril 2010. Bien que le format du diaporama ait été choisi pour la lecture en mobilité et que l'essor de cette dernière dans les transports urbains ait encouragé l'idée de publier un feuilleton quotidien du lundi au vendredi, les auteurs n'ont jamais décliné leur publication autrement que sur un site web conçu pour la consultation depuis un ordinateur personnel. En effet, les restrictions morales appliquées aux contenus de l'Appstore d'Apple ont découragé les auteurs d'investir dans des développements dédiés aux mobiles alors que leur récit exposait ponctuellement des scènes à caractère sexuel.

Malgré la divergence entre les représentations initiales des auteurs et les conditions de mise en œuvre de leur projet, les lecteurs sont parvenus à intégrer la pratique de cette lecture nouvelle à leurs usages. Cette réussite s'explique par le partage de représentations compatibles avec celles des auteurs pour trois autres cadres de référence : l'information en ligne, le feuilleton télévisé et la bande dessinée.

\section{L’information de presse en ligne, cadre de référence pour le modèle économique}

Les lecteurs qui ont plébiscité Les Autres Gens témoignent principalement d'une consultation matinale de la série à leur arrivée au bureau, ou bien le soir de retour à la maison. Cette consultation régulière dictée par le rythme de publication quotidien s'appuie sur des habitudes préexistantes de consultation de blogs en général (pas seulement ou par forcément de blogs BD), mais aussi de la presse en ligne.

\footnotetext{
de doctorat en Sciences de l'information et de la communication. Metz : Université de Lorraine. Disponible à l'adresse : http://docnum.univ-lorraine.fr/public/DDOC_T_2014_0112_FALGAS.pdf.

${ }^{9}$ Empruntant la notion de cadre à Erving Goffman (GOFFMAN, Erving, 1991. Les cadres de l'expérience. Trad de l'anglais par : JOSEPH, Isaac. Paris : Editions de Minuit. Le sens commun. ISBN 2707313726 9782707313720), Patrice Flichy définit le cadre de référence ainsi : " les acteurs d'une opération technique mobilisent tel cadre qui leur permet de percevoir, de comprendre les phénomènes auxquels ils assistent et d'organiser leurs propres actions » (FLICHY, Patrice, 1995. L'innovation technique: récents développements en sciences sociales, vers une nouvelle théorie de l'innovation. Paris : La Découverte. Sciences et société. ISBN 2707140007).
} 
II s'avère que le propos de la série Les Autres Gens situe l'action le jour même de sa publication. Le récit se déroule simultanément à l'actualité journalistique, dont la référence appuie la familiarité du lecteur avec les personnages qui - comme lui - vivent des périodes électorales ou le traumatisme causé par l'accident nucléaire de Fukushima.

La proximité du projet avec le champ de l'actualité ne s'arrête pas là : le scénariste et porteur du projet de la série y puise l'inspiration de son modèle économique. C'est en effet en tant que consommateur convaincu de sites d'information tels que Mediapart ou @rrêt sur image qu'il conçoit comme une évidence l'idée de commercialiser la série sous forme d'abonnement. Cette décision lui a valu des échanges houleux durant les premiers mois de publication avec les tenants d'une bande dessinée en ligne gratuite, marqués par le modèle du blog BD qui s'était popularisé depuis 2005. Le lectorat de la série s'est pour sa part constitué avec des lecteurs peu ou pas férus de bande dessinée ou de blog BD, mais plutôt coutumiers des usages liés aux pratiques informationnelles en ligne.

Avoir apporté la preuve de la viabilité de la formule de l'abonnement constitue aujourd'hui aux yeux du créateur-scénariste de la série sa principale contribution à l'évolution de la bande dessinée numérique. C'est d'ailleurs ce qu'en ont retenu les porteurs des projets de revues de bande dessinée numérique qui ont succédé à la série Les Autres Gens, au détriment de bien d'autres caractéristiques de la série.

\section{Le feuilleton télévisé, cadre de référence pour la forme narrative}

C'est autour de la référence partagée au cadre du feuilleton et du feuilleton télévisé tel que l'ont popularisé les séries américaines des dernières années que le créateur-scénariste de la série a réuni une centaine de collaborateurs pour dessiner ses personnages et l'aider dans l'écriture des dialogues. C'est aussi cette référence qui s'est révélée déterminante de la capacité de certains lecteurs à se passionner pour la lecture de la série au point d'en débattre entre eux sur le forum officiel comme sur Twitter, comme peuvent le faire les hardcore fans des séries télévisées américaines ${ }^{10}$. Aucun élément ne laisse supposer que l'ancrage dans la tradition de la bande dessinée ait pu avoir un impact comparable sur la constitution d'une communauté de fans autour de la série.

\section{La bande dessinée, cadre de référence esthétique et pour la collaboration entre les auteurs}

Jamais un projet de bande dessinée n'avait réuni autant d'auteurs. Or l'auteur de bande dessinée de la tradition franco-belge puise son identité dans la reconnaissance de son style et de sa signature. II était inconcevable d'obtenir l'adhésion de suffisamment de collaborateurs autour de ce projet en exigeant une organisation en studio. C'est ainsi que chaque épisode de la série représente graphiquement les personnages selon la sensibilité et le style de celui qui le met en image. Pour chaque auteur, la contribution à la série relevait d'une collaboration somme toute classique entre un dessinateur et un scénariste, collaboration qui était assortie du versement forfaitaire de droits pour la publication sur le site web et de la perspective d'autres retombées financières en cas d'exploitation sur d'autres supports numériques ou imprimés. De cette manière et grâce aux outils de travail collaboratifs disponibles sur Internet, le créateur-scénariste est parvenu à organiser la publication sans discontinuer durant deux ans et demi. L'identité de chacun de ses collaborateurs en tant qu'auteur de bande dessinée était non seulement préservée, mais même renforcée : les dimensions et la singularité de la série Les Autres Gens en ont fait un véritable catalogue de la jeune génération d'auteurs de bande dessinée. Les lecteurs ne s'y

\footnotetext{
${ }^{10}$ La notion de hardcore fan est empruntée à Henri Jenkins. Il désigne ainsi les fans qui démontrent des pratiques créatives, contributives ou participatives autour de l'objet de leur passion. JENKINS, Henry, 2006. Convergence culture: where old and new media collide. New York : New York University Press. ACLS Humanities E-Book. ISBN 9780814742815.
} 
FALGAS, Julien, 2016. Pour une Sociologie des usages et de l'innovation appliquée aux récits innovants.

In : ROBERT, Pascal, Bande dessinée et numérique. CNRS Editions, collection Les Essentiels d'Hermès. Paris. p. 135-153.

sont pas trompés : certains ont découvert la série par curiosité pour la contribution de l'un de leurs auteurs favoris et beaucoup y ont découvert des auteurs dont ils ont eu à cœur de lire d'autres œuvres.

Loin de tirer sa source de l'ambition d'invention vis-à-vis d'une tradition donnée, l'innovation narrative exige de se conformer à des cadres préexistants dont le choix et l'assemblage se révèlent originaux. La réussite d'une telle innovation repose sur la reconnaissance puis l'adoption par les publics des cadres de référence choisis et assemblés par les auteurs. L'adhésion des publics est facilitée par le fait que les auteurs ont choisi et assemblé leurs cadres de référence avec spontanéité, guidés par leur ambition narrative.

\section{MediaEntity, récit d'une production de sens ${ }^{11}$ autour du turbomédia}

MediaEntity vise certes à offrir une expérience de récit transmédia, mais sa première expression est une bande dessinée numérique. La publication des quatre épisodes de ce "pilote" est scénarisée sous la forme d'un jeu de piste organisé par courrier électronique et sur la page Facebook de MediaEntity : les lecteurs désireux de lire le prochain épisode avant sa parution publique sont invités à résoudre des énigmes à l'issue desquelles un code d'accès leur est délivré. C'est un des personnages de la fiction qui est l'avatar des auteurs dans ces échanges. Sur le site web de la série, les épisodes revendiquent le format " turbomédia » et plusieurs autres expressions de l'univers sont annoncées : adaptation imprimée de la bande dessinée, docufiction, websérie vidéo participative, jeu de rôles, application mobile, nouvelles littéraires... Les dispositifs transmédias sont de plus en plus courants autour de films de cinéma ou de séries télévisées, mais c'est la première fois qu'un tel dispositif est développé de manière aussi aboutie autour d'une bande dessinée.

Les auteurs de MediaEntity ne sont que deux : un scénariste et une dessinatrice. La publication de la série est le résultat d'un projet de longue date. Le projet a débuté par une bourse d'écriture financée dans le cadre d'un dispositif public destiné à encourager la création de séries télévisées françaises. II s'est poursuivi dans le cadre d'un programme de recherche et développement transmédia porté par l'opérateur de télécommunications Orange. Ce programme n'ayant pas débouché sur une production, et malgré les opportunités qui s'offraient à eux, les auteurs de MediaEntity ont préféré se détourner du monde audiovisuel. Leur sentiment était que ce secteur risquait de leur confisquer l'univers qu'ils avaient imaginé et qu'ils n'auraient pas toute liberté pour l'aborder comme ils l'entendaient. La bande dessinée revêtait à leurs yeux l'image d'un secteur moins castrateur, du fait d'une économie moins gourmande. La création de bande dessinée leur paraissait également accessible sans moyens supplémentaires, là où une production audiovisuelle n'était pas concevable sans être accompagnés de nombreux collaborateurs. On retrouve là un élément essentiel du cadre de référence à la bande dessinée, tel que l'avaient déjà exprimés les auteurs et les lecteurs de la série Les Autres Gens : la bande dessinée désigne aux yeux de ces auteurs le mode d'expression narratif et graphique le plus économe en moyens, et également le plus ouvert et le plus souple.

Ne parvenant pas à conclure de contrat éditorial compatible avec leurs ambitions transmédia, les auteurs décident de s'autopublier sur Internet. Le web revêt pour eux, comme pour le créateur-scénariste de la série Les Autres Gens, l'image d'un eldorado où tout serait possible. Ils sont confortés dans cette idée

\footnotetext{
${ }^{11}$ Cette notion est empruntée aux sciences de gestion, elle s'appuie sur le travail de Karl E. Weick et en particulier à son ouvrage WEICK, Karl E., 1995. Sensemaking in organizations. Thousand Oaks : Sage Publications. Foundations for organizational science. ISBN 0803971761.

Selon Dominique Maurel, le modèle de sensemaking proposé par Karl E. Weick mérite d'être davantage mis à profit en sciences de I'information et de la communication : MAUREL, Dominique, 2010. Sense-making: un modèle de construction de la réalité et d'appréhension de l'information par les individus et les groupes. In : Études de communication. 2010. Vol. 2/2010, n 35, p. 31 --46.
} 
par le fait que la dessinatrice est l'auteure d'un clip musical totalisant plusieurs millions de vues sur Youtube. Les auteurs de MediaEntity ne connaissaient alors pas encore le format «turbomédia ». Tout comme les auteurs de la série Les Autres Gens, ils envisagent spontanément une lecture case à case inspirée par l'usage des smartphones, bien qu'ils ne possèdent ni l'un ni l'autre un tel équipement. Ainsi, les auteurs des deux récits ont-ils fortement appuyé leurs projets respectifs sur des représentations nourries de leur imaginaire pour les nouvelles technologies, à partir de l'extrapolation d'expériences et d'informations relativement ténues.

Lorsque le scénariste découvre le "turbomédia », la dessinatrice et lui-même sont conquis. Pour eux, ce format narratif conjugue à merveille ce qui les avait poussés à s'orienter vers la bande dessinée tout en répondant à leur culture de l'audiovisuel. En effet, la dessinatrice est issue de formations en film d'animation, or l'inventeur du « turbomédia » (Balak) tout comme son principal promoteur (Malec) sont des professionnels du storyboard de dessin animé. Ceci explique que l'appropriation de ce format narratif se soit révélée immédiate pour des auteurs issus de cette même culture, mais aussi pour leurs lecteurs. Ces derniers ignorent tout du «turbomédia ", ils y reconnaissent pourtant les influences de la bande dessinée et de l'audiovisuel tout en saluant une forme narrative nouvelle et convaincante.

Quelques semaines après la parution du quatrième épisode de MediaEntity, à l'occasion du Festival International de la Bande Dessinée 2012 à Angoulême, les auteurs signent un contrat d'édition avec les Editions Delcourt. L'ancrage du récit dans une forme reconnue de bande dessinée numérique n'est pas étranger à cette réussite. Le scénariste comme la dessinatrice avaient toujours affirmé que la forme numérique de leur récit et ses perspectives transmédias primait sur son adaptation imprimée. Pourtant, rattrapés par la temporalité de la production d'un album de bande dessinée et les contraintes économiques, leur nouvelle identité professionnelle d'auteurs de bande dessinée s'impose à eux sur leurs préférences artistiques. Suite à l'expérimentation peu concluante d'une commercialisation de la version «turbomédia » avec ComiXology, il faudra attendre la parution du troisième album en juin 2015 pour découvrir l'intégralité de la version «turbomédia » du deuxième album sur le site Internet de la série $^{12}$.

Le «turbomédia » a joué le rôle de trait d'union entre le monde de l'audiovisuel et celui de la bande dessinée : issus de l'audiovisuel et attirés par la bande dessinée, les auteurs de MediaEntity ont trouvé dans ce format hybride un moyen d'intégrer le monde de la bande dessinée. Après avoir rejoint le monde de l'art de la bande dessinée, ils ont toutefois dû se conformer à ses attentes au point de faire passer au second plan ce qui constituait à leurs yeux la forme la plus authentique de leur récit.

Le potentiel d'innovation d'une nouvelle forme narrative est dépendant de sa capacité à s'intégrer dans une dynamique économique à même d'assurer sa subsistance matérielle et l'identité professionnelle de ses auteurs.

\footnotetext{
${ }^{12}$ Information recueillie auprès de Yannick Lejeune, éditeur de MediaEntity chez Delcourt, à l'occasion du débat professionnel « du papier au turbomédia » organisé à Marne-la-Vallée le 11 avril 2015 dans le cadre du PULP Festival.
} 


\section{L’héritage de la bande dessinée}

Les définitions de la bande dessinée en tant que forme narrative sont certes bousculées par les nouvelles productions numériques. Pourtant leurs auteurs et leurs lecteurs y reconnaissent sans mal l'essence de leurs représentations de la bande dessinée en tant qu'être culturel ${ }^{13}$.

Dans les réponses et les témoignages des auteurs et des lecteurs interrogés, la bande dessinée apparaît comme :

- l'expression de l'ambition narrative partagée entre quelques auteurs (à la différence d'une création audiovisuelle collective dirigée par un réalisateur),

- incarnée sous une forme graphique (à la différence d'un texte littéraire ou d'un récit oral) qui autorise une relative économie de moyens.

Sur la base de ces fondements solides, les productions numériques identifiées par leurs auteurs et leurs lecteurs comme des "bandes dessinées numériques " semblent pouvoir s'autoriser tous les emprunts, citations et détournements d'autres cadres de référence.

$\mathrm{Au}$ travers de ses prolongements numériques, la bande dessinée moderne perpétue l'héritage polygraphique dont elle a été légataire tout au long du $X X^{e}$ siècle et démontre qu'elle n'a pas perdu sa capacité à dialoguer avec les technologies contemporaines ${ }^{14}$. La seule limite à la pérennité de cette inventivité semble résider dans l'appareil économique sur lequel se fonde l'identité professionnelle des auteurs.

\section{Glossaire}

Cadres de référence : notion de sociologie empruntée à Erving Goffman (GOFFMAN, Erving, 1991. Les cadres de l'expérience. Trad de l'anglais par: JOSEPH, Isaac. Paris: Editions de Minuit. Le sens commun. ISBN 2707313726 9782707313720) par Patrice Flichy et qu'il définiti ainsi : « les acteurs d'une opération technique mobilisent tel cadre qui leur permet de percevoir, de comprendre les phénomènes auxquels ils assistent et d'organiser leurs propres actions » (FLICHY, Patrice, 1995. L'innovation technique: récents développements en sciences sociales, vers une nouvelle théorie de l'innovation. Paris : La Découverte. Sciences et société. ISBN 2707140007).

Hardcore fans : Henri Jenkins désigne ainsi les fans qui démontrent des pratiques créatives, contributives ou participatives autour de l'objet de leur passion (JENKINS, Henry, 2006. Convergence culture: where old and new media collide. New York : New York University Press. ACLS Humanities E-Book. ISBN 9780814742815).

Production de sens : chez Karl E. Weick, la production de sens est le processus permettant de savoir ce que l'on sait avant même d'en avoir fait l'expérience sensible (WEICK, Karl E., 1995. Sensemaking in organizations. Thousand Oaks : Sage Publications. Foundations for organizational science. ISBN 0803971761). «Weick identifie sept caractéristiques du sense- making : celui-ci se veut un processus ancré dans la construction de l'identité, rétrospectif puisqu'il n'est possible de bien comprendre une

\footnotetext{
${ }^{13}$ Cette notion est empruntée à Yves Jeanneret qui désigne ainsi les idées et les objets qui se chargent de valeur en circulant au travers des "carrefours de la vie sociale". Cf. JEANNERET, Yves, 2008. Penser la trivialité Volume 1: la vie triviale des êtres culturels. Paris : Hermès science publications; Lavoisier. Communication, médiation et construits sociaux. ISBN 9782746218789.

${ }^{14}$ SMOLDEREN, Thierry, 2009. Naissances de la bande dessinée: de William Hogarth à Winsor McCay [en ligne]. Bruxelles : les Impressions nouvelles. ISBN 9782874490828.
} 
action qu'après l'avoir posée, générateur d'environnements modelés par les acteurs organisationnels, social car il s'inscrit dans les valeurs et interprétations partagées par un groupe d'individus, continu et évolutif, basé sur des signaux et des indices et, enfin, vraisemblable pour les acteurs organisationnels » (MAUREL, Dominique, 2010. Sense-making: un modèle de construction de la réalité et d'appréhension de l'information par les individus et les groupes. In : Études de communication. 2010. Vol. 2/2010, n 35, p. 31--46).

Récit transmédia : Henri Jenkins désigne ainsi tout récit-monde que les publics appréhendent de manière parcellaire en accédant à tout ou partie des formes narratives proposées. Le contact d'un univers fictionnel par le biais d'une pluralité de médias entraîne un effet de réel, du fait que notre appréhension de la réalité s'appuie elle-même pour bonne part sur sa perception au travers de ces mêmes médias (JENKINS, Henry, 2006. Convergence culture: where old and new media collide. New York : New York University Press. ACLS Humanities E-Book. ISBN 9780814742815).

Turbomédia: format narratif inventé par Yves Bigerel sous le pseudonyme de Balak et promu par Alexandre Ulmann sous le pseudonyme de Malec. L'auteur expose son récit sous la forme d'une succession de diapositives que le lecteur consulte au rythme de sa lecture. De nature graphique et pouvant comporter de textes ou des phylactères sur le modèle de la bande dessinée, les diapositives peuvent intégrer du contenu animé, sonore ou interactif. Le recours à des effets cinétiques inspirés de la pratique du storyboard d'animation est caractéristique des productions turbomédia. Une frise interactive retrace l'histoire de ce format narratif entre 2009 et 2014 : Mast et Geoffo, 2014, 5 ans de Turbomédia, disponible en ligne : http://ybrik-media.com/bon-anniversaire-le-turbomedia/ (consultée le 23 janvier 2015). 